2009

\title{
Discovery Of The Energetic Pulsar J1747-2809 In The Supernova Remnant G0.9+0.1
}

F. Camilo

S. M. Ransom

B. M. Gaensler

D. R. Lorimer

Follow this and additional works at: https://researchrepository.wvu.edu/faculty_publications

\section{Digital Commons Citation}

Camilo, F.; Ransom, S. M.; Gaensler, B. M.; and Lorimer, D. R., "Discovery Of The Energetic Pulsar J1747-2809 In The Supernova Remnant G0.9+0.1" (2009). Faculty Scholarship. 10.

https://researchrepository.wvu.edu/faculty_publications/10 


\title{
DISCOVERY OF THE ENERGETIC PULSAR J1747-2809 IN THE SUPERNOVA REMNANT G0.9+0.1
}

\author{
F. CAMilo ${ }^{1}$, S. M. RANSOM ${ }^{2}$, B. M. GAENSLER ${ }^{3}$, AND D. R. LORIMER ${ }^{4}$ \\ ${ }^{1}$ Columbia Astrophysics Laboratory, Columbia University, New York, NY 10027, USA \\ 2 National Radio Astronomy Observatory, Charlottesville, VA 22903, USA \\ 3 Sydney Institute for Astronomy, School of Physics, The University of Sydney, NSW 2006, Australia \\ ${ }^{4}$ Department of Physics, West Virginia University, Morgantown, WV 26506, USA \\ Received 2009 May 21; accepted 2009 June 17; published 2009 June 30
}

\begin{abstract}
The supernova remnant G0.9+0.1 has long been inferred to contain a central energetic pulsar. In observations with the NRAO Green Bank Telescope at $2 \mathrm{GHz}$, we have detected radio pulsations from PSR J1747-2809. The pulsar has a rotation period of $52 \mathrm{~ms}$, and a spin-down luminosity of $\dot{E}=4.3 \times 10^{37} \mathrm{erg} \mathrm{s}^{-1}$, the second largest among known Galactic pulsars. With a dispersion measure of DM $=1133 \mathrm{pc} \mathrm{cm}^{-3}$, PSR J1747-2809 is distant, at $\approx 13 \mathrm{kpc}$ according to the NE2001 electron density model, although it could be located as close as the Galactic center. The pulse profile is greatly scatter-broadened at a frequency of $2 \mathrm{GHz}$, so that it is effectively undetectable at $1.4 \mathrm{GHz}$, and is very faint, with period-averaged flux density of $40 \mu \mathrm{Jy} \mathrm{at} 2 \mathrm{GHz}$.
\end{abstract}

Key words: ISM: individual (G0.9+0.1) - pulsars: individual (PSR J1747-2809) - stars: neutron

\section{INTRODUCTION}

With a Galactic core-collapse supernova rate of $1-3$ per century (e.g., Diehl et al. 2006), young neutron stars (with age $\lesssim 10 \mathrm{kyr}$ ) are rare. Nevertheless, even by those standards the known sample is woefully incomplete. Only 12 rotationpowered pulsars with characteristic age $\tau_{c}<10 \mathrm{kyr}$ are known in the Galaxy, and less than 20 such pulsar-supernova remnant (SNR) associations are firmly established. Developing a more complete picture of the young pulsar population contributes to an understanding of the birthrate of neutron stars and of the physics of their creation in stellar core collapses.

While most SNRs are in principle good locations to search for young pulsars, wholesale searches require multiple telescope pointings each with potentially inadequate sensitivity and have had limited success (e.g., Gorham et al. 1996; Kaspi et al. 1996; Lorimer et al. 1998). A more recent and successful approach has been to target, with single deep observations, compact pulsar wind nebulae (PWNe), identified via X-ray or radio imaging and spectroscopy, which indicate the presence of a young neutron star even in the absence of the detection of pulsations (e.g., Camilo et al. 2002a, 2002b, 2002c, 2006; Halpern et al. 2001; Roberts et al. 2002). Detection of the period $P, P$, and derived quantities, underlies significant further understanding of the pulsar, its relativistic wind, PWN, and environment (see, e.g., Gaensler \& Slane 2006).

The composite SNR G0.9+0.1 consists of a radio shell 8' in diameter surrounding a $2^{\prime}$ PWN (Helfand \& Becker 1987; see Figure 1). Based on the very high interstellar absorption $\left(N_{H} \approx 1.3 \times 10^{23} \mathrm{~cm}^{-2}\right.$; Gaensler et al. 2001; Porquet et al. 2003; Sidoli et al. 2000, 2004), its distance is large, here parameterized by $d_{10}=d /(10 \mathrm{kpc})$. The PWN is luminous in radio, with $L_{r}\left(10^{7}-10^{12.4} \mathrm{~Hz}\right.$ ) $=1.7 \times 10^{35} d_{10}^{2} \mathrm{erg} \mathrm{s}^{-1}$ (Dubner et al. 2008), and filled with X-ray synchrotron emission with $L_{X}(2-10 \mathrm{keV})=0.4 L_{r}$ (Porquet et al. 2003). It is also a very-high-energy $\gamma$-ray source, with $L_{\gamma}(>0.2 \mathrm{TeV})=0.4 L_{X}$ (Aharonian et al. 2005). Based on these energetics and a variety of empirical relations, it has been predicted that the pulsar powering this $\mathrm{PWN}$ has $P \sim 0.1-0.2 \mathrm{~s}$ and spin-down luminosity $\dot{E} \sim 2 \times 10^{37} \mathrm{erg} \mathrm{s}^{-1}$ (Dubner et al. 2008; Mattana et al. 2009; Sidoli et al. 2000), while the SNR shell size implies an age of about 1-7 kyr (Mereghetti et al. 1998). The hard X-ray point source CXOU J174722.8-280915, with $1 \%$ the luminosity of the PWN and surrounded by small-scale ordered structure, is likely emission from the pulsar (Gaensler et al. 2001). We have searched this location for a radio pulsar, and in this Letter report the discovery of PSR J1747-2809, the central pulsar in SNR G0.9+0.1.

\section{OBSERVATIONS AND RESULTS}

We began our search for the pulsar in SNR G0.9+0.1 at the ATNF Parkes telescope, where during 2002-2005 we used three combinations of search frequencies and filterbank data acquisition systems to do four very long observations (see Table 1). The Cordes \& Lazio (2002) NE2001 free electron distribution model predicts that in this direction, for $d_{10}=1$, the expected dispersion measure would be $\mathrm{DM}=750 \mathrm{pc} \mathrm{cm}^{-3}$, and that the pulse broadening due to interstellar scattering at the standard search frequency of $1.4 \mathrm{GHz}$ would be $\tau_{1.4} \gtrsim 10 \mathrm{~ms}$. Given the possibility of larger actual DM and $\tau_{1.4}$, one of our observations was at $3 \mathrm{GHz}$.

We analyzed all search data with standard pulsar search techniques implemented in PRESTO (Ransom 2001; Ransom et al. 2002), including the excision of radio frequency interference (RFI) and a nearly optimal set of trial DMs (for more details see, e.g., Camilo et al. 2006). We dedispersed at up to twice the maximum Galactic DM of $1580 \mathrm{pc} \mathrm{cm}^{-3}$ predicted by NE2001 in this direction. No new pulsars were detected in any of the Parkes data sets.

The Parkes sensitivity limits at $1.4 \mathrm{GHz}$ correspond to luminosity $L_{1.4} \equiv S_{1.4} d^{2} \lesssim 7 d_{10}^{2} \mathrm{mJy} \mathrm{kpc}^{2}$, provided that scattering was not the limiting factor. At $3 \mathrm{GHz}$, although the sky background temperature was reduced, any ordinary pulsar would have had an even more significantly reduced flux, and the equivalent luminosity limit was worse. Since young pulsars can have luminosities at least as small as $L_{1.4} \approx 0.5 \mathrm{mJy} \mathrm{kpc}^{2}$ (Camilo et al. 2002d), we did a deeper search at the NRAO Green Bank Telescope (GBT).

In 2006 January, we observed G0.9+0.1 at the GBT at a central frequency of $1.95 \mathrm{GHz}$ using the Spigot autocorrelation spectrometer (Kaplan et al. 2005). For a typical spectral index 
Table 1

Observations of G0.9+0.1 and PSR J1747-2809

\begin{tabular}{|c|c|c|c|c|c|c|}
\hline Date & Telescope & $\begin{array}{c}\text { Frequency } \\
(\mathrm{GHz})\end{array}$ & $\begin{array}{c}\text { Bandwidth } \\
(\mathrm{MHz})\end{array}$ & $\begin{array}{l}\text { Sample Time } \\
(\mathrm{ms})\end{array}$ & $\begin{array}{c}\text { Integration Time } \\
\text { (hr) }\end{array}$ & $\begin{array}{c}P^{\mathrm{a}} \\
(\mathrm{ms})\end{array}$ \\
\hline 2002 May 16 & Parkes & 1.4 & $512 \times 0.5$ & 2.0 & 9.3 & $\ldots$ \\
\hline 2002 Nov 5 & Parkes & 1.4 & $512 \times 0.5$ & 1.6 & 7.5 & $\ldots$ \\
\hline 2003 Nov 5 & Parkes & 2.9 & $192 \times 3.0$ & 0.25 & 5.6 & $\ldots$ \\
\hline 2005 Oct 20 & Parkes & 1.4 & $96 \times 3.0$ & 0.25 & 9.4 & $\ldots$ \\
\hline 2006 Jan 8 & GBT & 1.9 & $768 \times 0.78$ & 0.08 & 6.0 & $52.137293(2)$ \\
\hline 2009 Mar 11 & GBT & 2.0 & $512 \times 1.56$ & 0.16 & 5.8 & $52.152855(2)$ \\
\hline
\end{tabular}

Note. ${ }^{\text {a }}$ The uncertainties in barycentric periods, given on the last digit in parentheses, are the $1 \sigma$ values obtained from TEMPO fits.

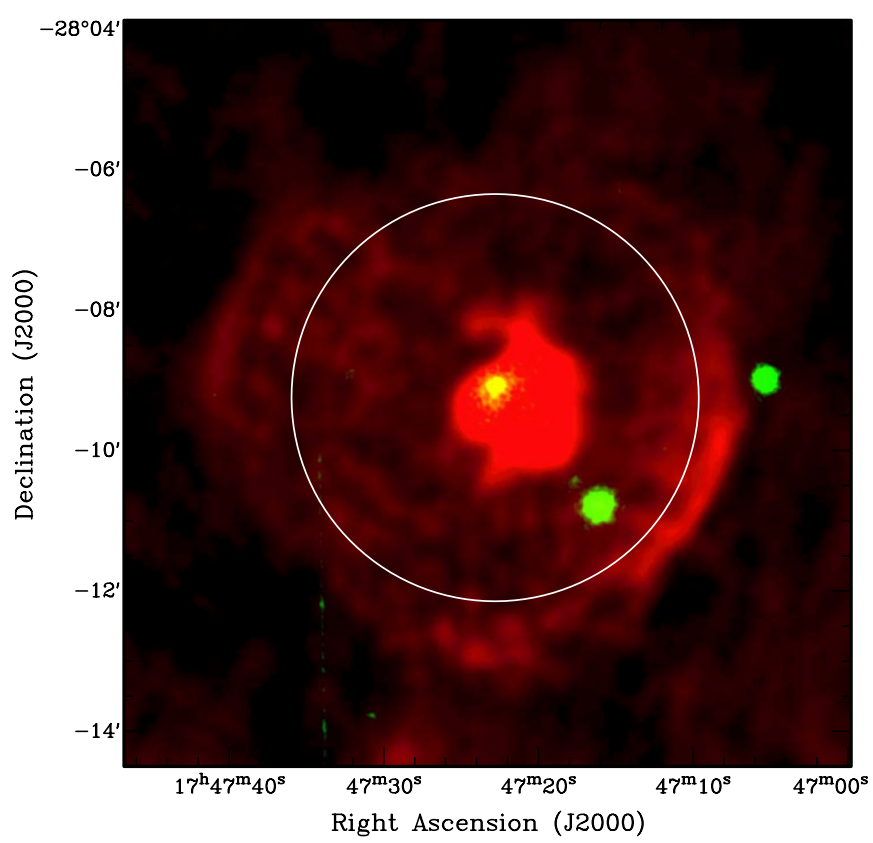

Figure 1. Composite radio and X-ray image of SNR G0.9+0.1 and its central PWN. In red is shown a $1.5 \mathrm{GHz}$ image, using VLA data taken in 1984 (reproduced from Gaensler et al. 2001); in green is displayed a $0.5-10 \mathrm{keV}$ $\mathrm{X}$-ray image using the EPIC PN detector on XMM-Newton, produced from an archival observation taken in 2003 (see Sidoli et al. 2004). Yellow denotes the radio- and X-ray-bright core of the PWN, within which is located the hard Xray point source CXOU J174722.8-280915 (Gaensler et al. 2001) that likely is emission from PSR J1747-2809. The VLA data are at an angular resolution of $15^{\prime \prime} \times 11^{\prime \prime}$, while the XMM-Newton data have been smoothed with a $5^{\prime \prime}$ Gaussian. The white circle shows the half-power point of the GBT beam for the pulsar search at $2 \mathrm{GHz}$. The X-ray source inside the SNR shell and to the southwest of the X-ray/radio core is the X-ray and $\gamma$-ray transient XMMU J174716.1-281048 = IGR J17464-2811 (Del Santo et al. 2007). The X-ray source outside and to the west of the SNR shell is coincident with the Chandra source CXO J174705.4-280859 (Muno et al. 2006).

of $\approx-1.6$ (Lorimer et al. 1995), the pulsar would be fainter by a factor of about 1.7 by comparison with $1.4 \mathrm{GHz}$. However, this was more than made up for by the larger gain and bandwidth at the GBT, such that our observation was a factor of about 2 more sensitive than the best Parkes search, both compared at the same fiducial frequency of $1.4 \mathrm{GHz}$. In addition, by using a higher search frequency, the scattering timescale was reduced by a factor of about 4 , which proved crucial in light of the actual pulsar period and the amount of observed scattering.

We identified in these data a highly dispersed $(\mathrm{DM}=1145 \mathrm{pc}$ $\mathrm{cm}^{-3}$ ) and scattered pulsar candidate with period $P=52 \mathrm{~ms}$ (Figure 2). It is a faint signal, with flux density $S_{2}=45 \mu \mathrm{Jy}$, compared to the detection threshold in this observation for a pulsar with these characteristics of approximately $20 \mu \mathrm{Jy}$. We confirmed the pulsar in an equivalent observation using the Green
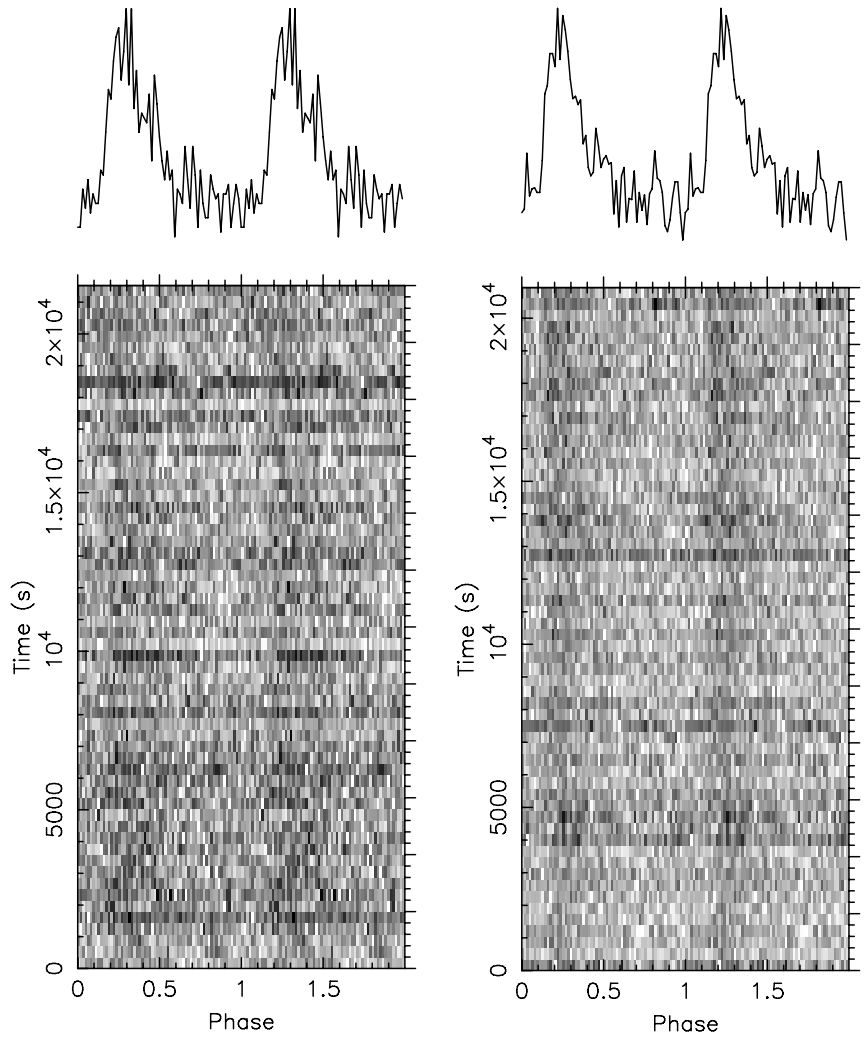

Figure 2. Detections of PSR J1747-2809 at GBT. Each $2 \mathrm{GHz}$ profile ( $P=$ $52 \mathrm{~ms}$ ) is repeated in phase, and is shown as a function of time (bottom) and as the sum of more than 400,000 pulses (top). Left: discovery observation from 2006 January 8 with Spigot. Right: confirmation observation from 2009 March 11 with GUPPI. The observed pulse FWHM increases from $0.14 P$ at $2.35 \mathrm{GHz}$ to $0.30 P$ at $1.75 \mathrm{GHz}$, due to interstellar scattering.

Bank Ultimate Pulsar Processing Instrument (GUPPI) ${ }^{5}$ on 2009 March 11 (Figure 2), for which $S_{2}=35 \mu \mathrm{Jy}$. In the presence of RFI, GUPPI, an FPGA-based digital spectrometer, has vastly improved performance compared to Spigot. This proved vital, since the RFI environment has degraded enormously in the intervening three years.

To measure the amount of scattering that clearly affects the pulse profile of PSR J1747-2809 (Figure 2), we did a fit to the GUPPI profile simultaneously in seven $100 \mathrm{MHz}$ wide subbands, assuming that the scattering timescale $\tau_{v}$ scales with observing frequency as $v^{-\alpha}$ with $\alpha=4$ (see, e.g., Bhat et al. 2004). We obtain $\tau_{1}=(0.21 \pm 0.03) \mathrm{s}$, scaled to the usually reported frequency of $1 \mathrm{GHz}$ (for $\alpha$ in the range 3.6-4.4, $\tau_{1}$ varies over $0.16-0.28 \mathrm{~s}$ ). This compares to $\tau_{1}=0.12 \mathrm{~s}$ predicted by Cordes \& Lazio (2002) for this DM and direction. The observed

\footnotetext{
5 https://wikio.nrao.edu/bin/view/CICADA/GUPPiUsersGuide
} 
DM is biased by the large degree of scattering, and in our fitting process we obtain the corrected $\mathrm{DM}=(1133 \pm 3) \mathrm{pc} \mathrm{cm}^{-3}$.

A comparison of the barycentric periods for the discovery and confirmation observations (Table 1) shows that the pulsar period increased by $\Delta P=(15.562 \pm 0.003) \mu \mathrm{s}$. The time interval between these observations was $\Delta T=1157.8$ days (MJD 54901.5-53743.7). The average period derivative is thus $\dot{P}=\Delta P / \Delta T=(1.5557 \pm 0.0003) \times 10^{-13}$. The corresponding derived pulsar parameters are $\dot{E}=4.3 \times 10^{37} \mathrm{erg} \mathrm{s}^{-1}, \tau_{c}=$ $P /(2 \dot{P})=5.3 \mathrm{kyr}$, and surface magnetic dipole field strength $B=2.9 \times 10^{12} \mathrm{G}$. For the observed DM, the NE2001 model yields $d \approx 13 \mathrm{kpc}$, though with substantial uncertainty.

PSR J1747-2809 has a magnetic field strength at its light cylinder of $B_{\mathrm{lc}}=1.9 \times 10^{5} \mathrm{G}$, which is the seventh highest among pulsars known in the Galactic disk. It has been suggested that $B_{\mathrm{lc}}$ is a controlling parameter for the emission of very narrow "giant" radio pulses (Cognard et al. 1996), and among the four pulsars known to emit giant pulses, PSR B0540-69, in the Large Magellanic Cloud (LMC), has the smallest value, $B_{\mathrm{lc}}=3.6 \times 10^{5} \mathrm{G}$. Therefore, in addition to the periodicity searches of the data listed in Table 1 (we also did a more sensitive folding analysis of all the Parkes data sets, using the subsequently determined values of $P$ and $\dot{P}$, but did not detect the pulsar), we carried out a search for dispersed individual pulses in the two GBT data sets. We used standard matched-filter techniques described in detail by Cordes \& McLaughlin (2003) and implemented in the SIGPROC pulsar search package. ${ }^{6}$ No single pulses were detected. However, our sensitivity was much degraded as a consequence of the large single-channel dispersion smearing (1-2 ms) and the huge scattering (about 13 ms at $2 \mathrm{GHz}$ ), but for which we could easily have detected giant pulses like those observed from PSR B0540-69 by Johnston \& Romani (2003).

\section{DISCUSSION}

PSR J1747-2809 is a rare young neutron star in that its spindown luminosity is surpassed among known Galactic pulsars only by the Crab. We have estimated the chance probability of detecting such a pulsar in our search by simulating an innerGalactic GBT survey with individual $6 \mathrm{hr}$ Spigot observations at $2 \mathrm{GHz}$. Considering the best population model of Lorimer et al. (2006), we find that such a survey would detect one ordinary (nonmillisecond) pulsar every $0.07 \mathrm{deg}^{2}$. Our beam area is $11 \%$ of this, which is also the probability of finding one such pulsar blindly. Until now, only the Crab was known with $\dot{E}>4 \times 10^{37} \mathrm{erg} \mathrm{s}^{-1}$ among 1600 ordinary Galactic pulsars. The probability of finding any such high $\dot{E}$ pulsar by chance is thus $\sim 0.11 / 1600=7 \times 10^{-5}$. PSR J1747-2809 is also very distant, based on its large DM, as expected for the pulsar in SNR G0.9+0.1 from the high $N_{H}$ to its PWN. The current positional uncertainty of PSR J1747-2809 is $\pm 3^{\prime}$, which is sufficient to locate it in projection within the G0.9+0.1 shell (Figure 1). Within this area, there are no radio or X-ray sources other than the G0.9+0.1 PWN that could plausibly account for a young pulsar with $\dot{E}=4 \times 10^{37} \mathrm{erg} \mathrm{s}^{-1}$, despite the great sensitivity of existing VLA, Chandra X-ray Observatory, and XMM-Newton observations. There is therefore no doubt that PSR J1747-2809 and $\mathrm{G} 0.9+0.1$ are associated.

The electron density model is not well constrained toward the distant inner Galactic regions (see Cordes \& Lazio 2002). Prior to the discovery of PSR J1747-2809, only nine pulsars

\footnotetext{
6 http://sigproc.sourceforge.net
}

were known with DM $>1100 \mathrm{pc} \mathrm{cm}^{-3}$, of which only two are within 20 degrees of the Galactic center (GC), none with independently estimated distances (Manchester et al. 2005). Therefore, the NE2001 distance of $13 \mathrm{kpc}$ for PSR J1747-2809 could be substantially in error. It is not excluded that the pulsar could be located at 8-9 kpc, physically near the GC. However, given both the very large $N_{H}$ and DM, it is unlikely that the pulsar is located substantially closer than the GC. Also, the ratio $N_{H} / \mathrm{DM} \approx 40$ for PSR J1747-2809 is higher than seen toward all but about four other pulsars (see Gaensler et al. 2004; Camilo et al. 2006). Gaensler et al. argue that this indicates a location behind substantial intervening molecular material, which would not be surprising for a location near or beyond the GC. It is also intriguing that two pulsars located only 0.3 degrees in projection from the GC (at a lateral distance of about $40 \mathrm{pc}$ if near it) have $\mathrm{DM} \approx 1100 \mathrm{pc} \mathrm{cm}^{-3}$ (Johnston et al. 2006), like PSR J1747-2809, which among known pulsars is the third nearest to the GC in projection. The scattering timescale for those two pulsars is a factor of a few larger than for PSR J1747-2809, but still very small compared to the levels expected if they were located within the scattering screen thought to surround the GC at a distance estimated as 50-330 pc by Lazio \& Cordes (1998). Johnston et al. (2006) therefore argue that those two pulsars are located somewhat in front of the GC. The same may apply to PSR J1747-2809, with a larger lateral separation of about 130 pc, but we also cannot exclude a substantially larger distance. We consider it likely that $0.8 \lesssim d_{10} \lesssim 1.6$.

The spin parameters of the new pulsar are similar to those of PSR J1833-1034 in SNR G21.5-0.9, which has $P=61 \mathrm{ms,}$ $\dot{E}=3.4 \times 10^{37} \mathrm{erg} \mathrm{s}^{-1}, \tau_{c}=4.9 \mathrm{kyr}$, and $d=4.7 \mathrm{kpc}$ (Camilo et al. 2006; Tian \& Leahy 2008). It is therefore of interest to compare the properties of both systems. The radius of the central PWN in G0.9+0.1 is $R_{\mathrm{PWN}}=3 d_{10}$ pc, nominally $50 \%$ larger than the PWN in G21.5-0.9. The radio luminosities may be similar, although this comparison is uncertain because of the much better frequency coverage for G21.5-0.9 (see Dubner et al. 2008; Bock et al. 2001, and references therein). In X-rays $(2-10 \mathrm{keV}), L_{X}(\mathrm{G} 0.9+0.1) \approx 0.7 d_{10}^{2} L_{X}(\mathrm{G} 21.5-0.9)$ (Porquet et al. 2003; Slane et al. 2000). At the highest energies, both PWNe are unresolved $\mathrm{TeV}$ sources, and colocated with their lower-energy counterparts, but G0.9+0.1 is $\approx 5 d_{10}^{2}$ times more luminous than G21.5-0.9 in a comparable band (Aharonian et al. 2005; Djannati-Atai et al. 2008; Gallant et al. 2008; Mattana et al. 2009).

Although the characteristic ages of both PSRs J1747-2809 and J1833-1034 are $\tau_{c}=5 \mathrm{kyr}$, the actual age of PSR J1833-1034 and its SNR is only $\tau \approx 1 \mathrm{kyr}$ (Bietenholz \& Bartel 2008; Bocchino et al. 2005; Camilo et al. 2006). PSR J1747-2809 could therefore be the older of the two, and one might be tempted to appeal to this possible difference to explain the larger size of the G0.9+0.1 PWN (and of its shell, whose radius $R_{\mathrm{SNR}}$ is also nominally $50 \%$ larger than that of G21.5-0.9) and (along with a smaller nebular magnetic field) its possibly lower X-ray efficiency. However, the nominal difference in the two efficiencies is small compared to the scatter observed among young pulsars. Dissimilar SN explosion kinetic energies and ejected masses could instead explain the different radii (see, e.g., van der Swaluw et al. 2001). Some observables point to the possibility that also for G0.9+0.1, $\tau<\tau_{c}$. The G0.9+0.1 shell is relatively bright in radio, unlike G21.5-0.9. This could be due to a different circumstellar environment, or may suggest that the SNR has swept enough mass and is transitioning to the adiabatic phase. Nevertheless, 
the PWN still retains approximate circular symmetry and a central location within the SNR shell, which likely indicates that a strong reverse shock has not yet formed. In that case, for the observed ratio $R_{\mathrm{PWN}} / R_{\mathrm{SNR}} \approx 0.25$ (similar to that of G21.5-0.9), the PWN evolutionary models of Blondin et al. (2001) indicate a small age. The PWN energetics also indicate a small age: for $\mathrm{G} 0.9+0.1$, the PWN magnetic and particle energies add to $\sim 10^{48} d_{10}^{2}$ erg (Dubner et al. 2008). This is smaller than $\dot{E} \tau_{c}=7 \times 10^{48} \mathrm{erg}$, suggesting that $\tau \ll \tau_{c}$ (see Chevalier 2005). All these ideas are outlined more fully for PSR J1833-1034/G21.5-0.9 by Camilo et al. (2006), including a discussion of the very significant differences with PSR J0205+6449 and its PWN 3C 58, despite comparable spin parameters. All these considerations point in the case of PSR J1747-2809/G0.9+0.1 to a system that, while not necessarily quite as young as PSR J1833-1034/G21.5-0.9, may have an age of no more than about 2-3 kyr. In turn, for spin evolution under constant magnetic moment with braking index in the observed range 2-3 (Livingstone et al. 2007), this would imply a birth period of $\gtrsim 40 \mathrm{~ms}$.

The TeV $\gamma$-ray emission observed from both G0.9+0.1 and G21.5-0.9 is most likely due to inverse Compton scattering of relativistic pulsar wind electrons. The seed photons for such scattering in general arise from dust, the cosmic microwave background, and star light. If PSR J1747-2809 is located close to the $\mathrm{GC}$, the main contribution to the photon field in G0.9+0.1 likely originates in star light (Aharonian et al. 2005), and this could account for much of the greater $\mathrm{TeV}$ luminosity of G0.9+0.1 compared to G21.5-0.9. If on the other hand, PSR J1747-2809 is located at a substantially greater distance, we would infer an even larger TeV luminosity for G0.9+0.1, while the stellar photon field would have lower energy density. Thus, careful modeling of the $\mathrm{TeV}$ emission from the G0.9+0.1 PWN may help to constrain the distance to the system. In any case, it may be that much of the observed difference between G0.9+0.1 and G21.5-0.9 arises not so much from intrinsic differences in the particle spectrum injected, respectively, by PSRs J1747-2809 and J1833-1034, as from their different environments.

The pulsars themselves have comparable luminosities and are very faint. For PSR J1833-1034, $L_{1.4} \approx 2 \mathrm{mJy} \mathrm{kpc}{ }^{2}$ and $L_{X} \approx 3 \times 10^{-5} \dot{E}$ (Camilo et al. 2006). For PSR J1747-2809, $L_{1.4} \approx 7 d_{10}^{2} \mathrm{mJy} \mathrm{kpc}^{2}$, and $L_{X} \approx 1.5 \times 10^{-5} d_{10}^{2} \dot{E}$, assuming that CXOU J174722.8-280915 is the counterpart (another potential candidate lies $10^{\prime \prime}$ to its north; Gaensler et al. 2001), which may be tested with the determination of a pulsar position via timing measurements. At $\mathrm{GeV}$ energies, pulsations have already been detected from PSR J1833-1034 with the Large Area Telescope on the Fermi Gamma-ray Space Telescope. ${ }^{7}$ PSR J1747-2809 is, at least, at twice the distance, and near the bright GC, and no detection by Fermi has yet been reported.

Because of interstellar scattering, PSR J1747-2809 is not detectable at $1.4 \mathrm{GHz}$ without a much more sensitive observation than is possible in practice $\left(\tau_{1.4} \approx 55 \mathrm{~ms}\right.$, estimated by scaling from $\left.\tau_{1}\right)$. Until recently, there were only four Galactic young pulsars known with $P<61 \mathrm{~ms}$ (the Crab, J1913+1011, B1951+32 in SNR CTB 80, and J2229+6114 associated with 3EG J2227+6122). The bright PSR J1410-6132, with $P=50 \mathrm{~ms}$ and $\mathrm{DM}=960 \mathrm{pc} \mathrm{cm}^{-3}$, but very scattered at $1.4 \mathrm{GHz}$ and discovered instead at $6 \mathrm{GHz}$ (O'Brien et al. 2008), and the similarly scattered but $\sim 100$ times fainter PSR J1747-2809, show

\footnotetext{
http://moriond.in2p3.fr/J09/transparents/caliandro.ppt
}

the importance of doing the utmost in dedicated searches of interesting objects to plumb the depths of the pulsar luminosity distribution and to minimize other biases against detecting short period neutron stars (see also Camilo et al. 2007; Johnston et al. 2006, for detection of other highly scattered pulsars). PSR J1747-2809 lies within the group of low-luminosity young pulsars $\left(L_{1.4} \lesssim 10 \mathrm{mJy} \mathrm{kpc}^{2}\right)$, nearly all of which were discovered in deep directed searches. These now make up one-third of all known young pulsars (see discussion in Camilo et al. 2006), and there is little reason to suppose that such pulsars are intrinsically rare. The future, if not bright, appears promising.

The GBT is operated by the National Radio Astronomy Observatory, a facility of the National Science Foundation operated under cooperative agreement by Associated Universities, Inc. We thank the staff at NRAO for quickly approving and scheduling the Rapid Response Science request to confirm the pulsar. The Parkes Observatory is part of the Australia Telescope, which is funded by the Commonwealth of Australia for operation as a National Facility managed by CSIRO. This work was supported in part by the NSF through grant AST-0908386. B.M.G. acknowledges the support of a Federation Fellowship from the Australian Research Council through grant FF0561298. D.R.L. is partially supported by West Virginia EPSCoR through a Research Challenge Grant.

Facilities: GBT (GUPPI, Spigot), Parkes (PMDAQ)

\section{REFERENCES}

Aharonian, F., et al. 2005, A\&A, 432, L25

Bhat, N. D. R., Cordes, J. M., Camilo, F., Nice, D. J., \& Lorimer, D. R. 2004, ApJ, 605,759

Bietenholz, M. F., \& Bartel, N. 2008, MNRAS, 386, 1411

Blondin, J. M., Chevalier, R. A., \& Frierson, D. M. 2001, ApJ, 563, 806

Bocchino, F., van der Swaluw, E., Chevalier, R., \& Bandiera, R. 2005, A\&A, 442, 539

Bock, D. C.-J., Wright, M. C. H., \& Dickel, J. R. 2001, ApJ, 561, L203

Camilo, F., Lorimer, D. R., Bhat, N. D. R., Gotthelf, E. V., Halpern, J. P., Wang, Q. D., Lu, F. J., \& Mirabal, N. 2002a, ApJ, 574, L71

Camilo, F., Manchester, R. N., Gaensler, B. M., Lorimer, D. L., \& Sarkissian, J. 2002b, ApJ, 567, L71

Camilo, F., Manchester, R. N., Gaensler, B. M., \& Lorimer, D. R. 2002c, ApJ, 579, L25

Camilo, F., Ransom, S. M., Gaensler, B. M., Slane, P. O., Lorimer, D. R., Reynolds, J., Manchester, R. N., \& Murray, S. S. 2006, ApJ, 637, 456

Camilo, F., Ransom, S. M., Halpern, J. P., \& Reynolds, J. 2007, ApJ, 666, L93 Camilo, F., et al. 2002d, ApJ, 571, L41

Chevalier, R. A. 2005, ApJ, 619, 839

Cognard, I., Shrauner, J. A., Taylor, J. H., \& Thorsett, S. E. 1996, ApJ, 457, L81

Cordes, J. M., \& Lazio, T. J. W. 2002, arXiv:astro-ph/0207156

Cordes, J. M., \& McLaughlin, M. A. 2003, ApJ, 596, 1142

Del Santo, M., Sidoli, L., Mereghetti, S., Bazzano, A., Tarana, A., \& Ubertini, P. 2007, A\&A, 468, L17

Diehl, R., et al. 2006, Nature, 439, 45

Djannati-Atai, A., De Jager, O. C., Terrier, R., Gallant, Y. A., \& Hoppe, S. 2008, in Proc. 30th ICRC, Vol. 2, ed. R. Caballero, J. D’Olivo, G. Medina-Tanco, L. Nellen, F. A. Sánchez, \& J. F. Valdés-Galicia (Mexico City: UNAM), 823 Dubner, G., Giacani, E., \& Decourchelle, A. 2008, A\&A, 487, 1033

Gaensler, B. M., Pivovaroff, M. J., \& Garmire, G. P. 2001, ApJ, 556, L107

Gaensler, B. M., \& Slane, P. O. 2006, ARA\&A, 44, 17

Gaensler, B. M., van der Swaluw, E., Camilo, F., Kaspi, V. M., Baganoff, F. K., Yusef-Zadeh, F., \& Manchester, R. N. 2004, ApJ, 616, 383

Gallant, Y. A., et al. 2008, in AIP Conf. Ser. 983, 40 Years of Pulsars: Millisecond Pulsars, Magnetars and More, ed. C. Bassa, Z. Wang, A. Cumming, \& V. M. Kaspi (Berlin: Springer), 195

Gorham, P. W., Ray, P. S., Anderson, S. B., Kulkarni, S. R., \& Prince, T. A. 1996, ApJ, 458, 257

Halpern, J. P., Camilo, F., Gotthelf, E. V., Helfand, D. J., Kramer, M., Lyne, A. G., Leighly, K. M., \& Eracleous, M. 2001, ApJ, 552, L125

Helfand, D. J., \& Becker, R. H. 1987, ApJ, 314, 203 
Johnston, S., Kramer, M., Lorimer, D. R., Lyne, A. G., McLaughlin, M., Klein, B., \& Manchester, R. N. 2006, MNRAS, 373, L6

Johnston, S., \& Romani, R. 2003, ApJ, 590, L95

Kaplan, D. L., et al. 2005, PASP, 117, 643

Kaspi, V. M., Manchester, R. N., Johnston, S., Lyne, A. G., \& D’Amico, N. 1996, AJ, 111, 2028

Lazio, T. J. W., \& Cordes, J. M. 1998, ApJ, 505, 715

Livingstone, M. A., Kaspi, V. M., Gavriil, F. P., Manchester, R. N., Gotthelf, E. V. G., \& Kuiper, L. 2007, Ap\&SS, 308, 317

Lorimer, D. R., Lyne, A. G., \& Camilo, F. 1998, A\&A, 331, 1002

Lorimer, D. R., Yates, J. A., Lyne, A. G., \& Gould, D. M. 1995, MNRAS, 273, 411

Lorimer, D. R., et al. 2006, MNRAS, 372, 777

Manchester, R. N., Hobbs, G. B., Teoh, A., \& Hobbs, M. 2005, AJ, 129, 1993

Mattana, F., et al. 2009, ApJ, 694, 12

Mereghetti, S., Sidoli, L., \& Israel, G. L. 1998, A\&A, 331, 77
Muno, M. P., Bauer, F. E., Bandyopadhyay, R. M., \& Wang, Q. D. 2006, ApJS, 165,173

O'Brien, J. T., et al. 2008, MNRAS, 388, L1

Porquet, D., Decourchelle, A., \& Warwick, R. S. 2003, A\&A, 401, 197

Ransom, S. M. 2001, PhD thesis, Harvard Univ.

Ransom, S. M., Eikenberry, S. S., \& Middleditch, J. 2002, AJ, 124, 1788

Roberts, M. S. E., Hessels, J. W. T., Ransom, S. M., Kaspi, V. M., Freire, P. C. C., Crawford, F., \& Lorimer, D. R. 2002, ApJ, 577, L19

Sidoli, L., Bocchino, F., Mereghetti, S., \& Bandiera, R. 2004, Mem. Soc. Astron. Ital., 75, 507

Sidoli, L., Mereghetti, S., Israel, G. L., \& Bocchino, F. 2000, A\&A, 361, 719

Slane, P., Chen, Y., Schulz, N. S., Seward, F. D., Hughes, J. P., \& Gaensler, B. M. 2000, ApJ, 533, L29

Tian, W. W., \& Leahy, D. A. 2008, MNRAS, 391, L54

van der Swaluw, E., Achterberg, A., Gallant, Y. A., \& Tóth, G. 2001, A\&A, 380,309 